\title{
Correction to: Usefulness of abbreviated breast MRI screening for women with a history of breast cancer surgery
}

\author{
Bo Hwa Choi ${ }^{1,3} \cdot$ Nami Choi $^{1}$ (D) Mi Young Kim ${ }^{1} \cdot J^{\prime}$ ung-Hyun Yang ${ }^{2} \cdot$ \\ Young Bum $\mathrm{Yoo}^{2} \cdot$ Hae Kyoung Jung ${ }^{4}$
}

Published online: 14 November 2017

(C) Springer Science+Business Media, LLC, part of Springer Nature 2017

Correction to: Breast Cancer Res Treat https://doi.org/10.1007/s10549-017-4530-z

In the original publication of the article, the acknowledgment section was missed out inadvertently. The acknowledgement section is below:

Acknowledgements This paper was written as part of Konkuk University's research support program for its faculty on sabbatical leave in 2015.

The original article can be found online at https://doi.org/10.1007/s10549-017-4530-z.

Nami Choi

nami0119@gmail.com

1 Department of Radiology, Konkuk University Medical Center, Konkuk University School of Medicine,

4-12 Hwayang-dong, Gwangjin-gu, Seoul 05030, Republic of Korea

2 Department of Surgery, Konkuk University Medical Center, Konkuk University School of Medicine, 4-12 Hwayang-dong, Gwangjin-gu, Seoul 05030, Republic of Korea

3 Department of Radiology, Gyeongsang National University School of Medicine and Gyeongsang National University Changwon Hospital, Changwon, Republic of Korea

4 Department of Radiology, CHA Bundang Medical Center, CHA University, Seongnam, Republic of Korea 